\title{
Hole Traps in ZnTe with CdTe Quantum Dots
}

\author{
E. Zielony ${ }^{a}$, E. PŁaczek-Popko ${ }^{a}$, Z. Gumienny ${ }^{a}$, J. Trzmiel ${ }^{a}$, \\ G. KARCZEWSKI ${ }^{b}$ AND M. GUZIEWICZ ${ }^{c}$ \\ ${ }^{a}$ Institute of Physics, Wrocław University of Technology, Wybrzeże Wyspiańskiego 27, 50-370 Wrocław, Poland \\ ${ }^{b}$ Institute of Physics, Polish Academy of Sciences, al. Lotników 32/46, 02-668 Warsaw, Poland \\ ${ }^{c}$ Institute of Electron Technology, al. Lotników 32/46, 02-668 Warsaw, Poland
}

\begin{abstract}
In this study the capacitance-voltage $(C-V)$ and deep level transient spectroscopy measurements have been performed on ZnTe ( $p$-type)-Ti/Al Schottky diodes containing a layer of CdTe self-assembled quantum dots and on the reference diodes without dots for comparison. Both kinds of investigated samples were grown by molecular beam epitaxy technique. The dots were formed during the Stransky-Krastanov growth mode. Comparison of the $C-V$ and deep level transient spectroscopy results obtained for both samples allows us to conclude that the $0.26 \mathrm{eV}$ trap observed exclusively for the QD sample can be assigned to some defects in a wetting layer or $\mathrm{CdTe} / \mathrm{ZnTe}$ interface.
\end{abstract}

PACS numbers: 73.61.Ga, 73.21.La, 73.20.Hb

\section{Introduction}

Semiconducting structures with quantum dots (QDs) have found an application in many electronic devices such as detectors of radiation, diode lasers, optical memories or single-electron transistors. It is well known that the presence of QDs within active region of the devices improves significantly their properties. This is directly related to the quantum effects arising from the confinement of electrons and holes in the quantum dots. In this context there is a need for understanding the charge carrier exchange between the QDs and their host material. The position of the quantum confined levels relative to the band edges of the host material can only be determined by electrical methods such as space charge capacitance techniques. Capacitance-voltage measurements $(C-V)$ and deep level transient spectroscopy (DLTS) have been proved to be powerful tools to probe the electronic properties of QD systems. DLTS allows investigation not only of the electronic level structure of the QDs, but also of the dynamics of emission and capture processes [1].

Zinc telluride ( $\mathrm{ZnTe}$ ) is a direct band gap semiconducting compound of $2.26 \mathrm{eV}$ energy gap at room temperature [2] and a high absorption coefficient of $10^{5} \mathrm{~cm}^{-1}$ [3]. For these properties it has been long considered as a very promising material for green optoelectronics. Actually, recently green light emitting diodes ZnTe based were realized [4]. With respect to these applications the $\mathrm{CdTe} / \mathrm{ZnTe}$ quantum dot systems are particularly interesting.

It is well known that the valence band offset for the $\mathrm{CdTe} / \mathrm{ZnTe}$ interface is of the order of $0.1 \mathrm{eV}[5,6]$ with the type II of alignment. Thus the confinement of holes is impossible. However it may be expected that the in- terface of the self-organized CdTe quantum dots embedded in ZnTe matrix would exhibit different properties for the strain accompanying QDs formation. In order to investigate the interface, the electronic properties of the $\mathrm{CdTe} / \mathrm{ZnTe} \mathrm{QD}$ system have been analyzed using $C-V$ and DLTS measurements.

\section{Experimental details}

Two types of samples with the same layer structure were processed by molecular beam epitaxy method: a reference sample without quantum dots and a sample with self-assembled quantum dots. The QD sample consists of $2 \mu \mathrm{m}$ thick $p^{+}$-type ZnTe:N buffer deposited on the $p$-type GaAs substrate, $0.7 \mu \mathrm{m} p$-type ZnTe:N, a layer of CdTe QDs and $0.11 \mu \mathrm{m}$ of $p$-type ZnTe:N cap (the same flux of $\mathrm{N}$ as in the case of the $0.7 \mu \mathrm{m}$ layer). The CdTe self-assembled QDs were grown in the StranskiKrastanov mode by deposition of three monolayers of CdTe. The presence of QDs was confirmed by photoluminescence measurements run at $T=10 \mathrm{~K}$.

The AuZnAu ohmic contacts were formed on the backside of GaAs substrate layer and Schottky $\mathrm{Ti}(5 \mathrm{~nm}) / \mathrm{Al}(200 \mathrm{~nm})$ contacts were deposited by the photolithography method on the ZnTe:N cap layer. The capacitance--voltage $(C-V)$ and DLTS measurements were performed on the lock-in based DLS-82E System (SEMITRAP, Hungary).

\section{Results and discussion}

In Fig. 1 the $C-V$ plots for both samples measured at room temperature are shown. The $C-V$ plot for the sample with QDs exhibits a characteristic step for forward 
bias, within the range of $0 \mathrm{~V}$ to $1 \mathrm{~V}$ (Fig. 1 ). It is not observed on the $C-V$ characteristics for the reference sample. This step presumably corresponds to the discharging of QDs for the following arguments. The room temperature $C-V$ measurements for the samples yield net acceptor concentration, $N_{\mathrm{A}}$, in the order of $5 \times 10^{15} \mathrm{~cm}^{-3}$. Such a value of $N_{\mathrm{A}}$ reveals the depletion width around $0.3 \mu \mathrm{m}$. The layer of CdTe QDs is located at a distance of $0.11 \mu \mathrm{m}$ below the $\mathrm{ZnTe}-\mathrm{Ti} / \mathrm{Al}$ interface — within the depletion region width. Therefore the discharging of the QDs would take place for forward bias. It is noteworthy to point out that the rectifying properties of the Schottky contacts with QDs were found to be better than those for the reference sample. The barrier height deduced from the $C-V$ measurements is higher for the reference sample $(1.6 \mathrm{eV})$ than for the QD sample $(0.5 \mathrm{eV})$ implying the presence of a thin insulating layer between the metal and ZnTe cap for this contact.

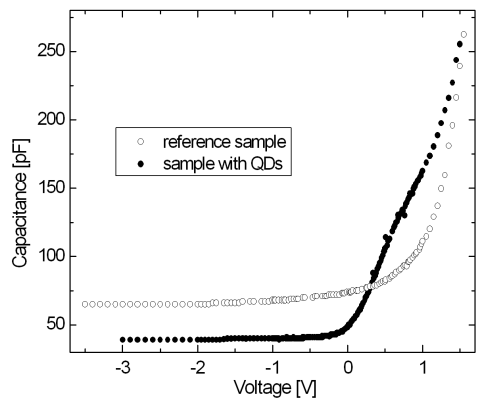

Fig. 1. $\quad C-V$ characteristics taken at $300 \mathrm{~K}$ for the sample with QDs (full dots) and for the reference sample (empty circles).

DLTS measurements were performed at temperatures in the range from $77 \mathrm{~K}$ to $300 \mathrm{~K}$ and lock-in frequencies in the range of $2-2500 \mathrm{~Hz}$ under dark conditions. Typical DLTS temperature scans for both samples performed at reverse bias equal to $-1.5 \mathrm{~V}$, with the filling pulse $U_{1}=0 \mathrm{~V}$ of $20 \mu$ s width at lock-in frequency equal to $1170 \mathrm{~Hz}$ are shown in Fig. 2. For the sample with quantum dots a broad DLTS signal peak appears with a low temperature shoulder. The position of the shoulder fits in to the position of the DLTS peak for the reference sample. On the other hand, for the latter one a broad high temperature signal can be noted. This signal is missing for the DLTS spectrum of the QD sample. The fact that this signal is very broad may indicate that it is linked with the continuum of states located at a poor $\mathrm{ZnTe}$-metal interface for the reference sample.

In Fig. 3 the Arrhenius plots corresponding to the major DLTS signal peaks observed for both samples obtained at $U_{\mathrm{R}}=-1.5 \mathrm{~V}$ and $U_{1}=0 \mathrm{~V}$ for different lock-in frequencies are presented. Thermal activation energy determined from the plot for the dominant hole trap in the reference sample equals to $0.16 \mathrm{eV}$ and capture cross-section $1.4 \times 10^{-16} \mathrm{~cm}^{2}$. This trap is commonly observed for $p$-type $\mathrm{ZnTe}$ and it is assigned to the $\mathrm{Zn}$ vacancy [7].

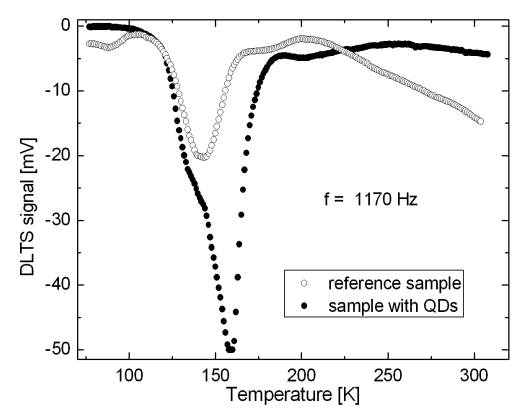

Fig. 2. DLTS spectra measured for both samples taken at reverse bias $U_{\mathrm{R}}=-1.5 \mathrm{~V}, U_{1}=0 \mathrm{~V}$. Lock-in frequency $f=1170 \mathrm{~Hz}$.

The trap probably also manifests itself as a low temperature shoulder in the QD DLTS spectrum. The Arrhenius plot of the dominant peak for the QDs sample reveals the thermal activation energy equal to $0.26 \mathrm{eV}$ and capture cross-section $\sigma=10^{-15} \mathrm{~cm}^{2}$.

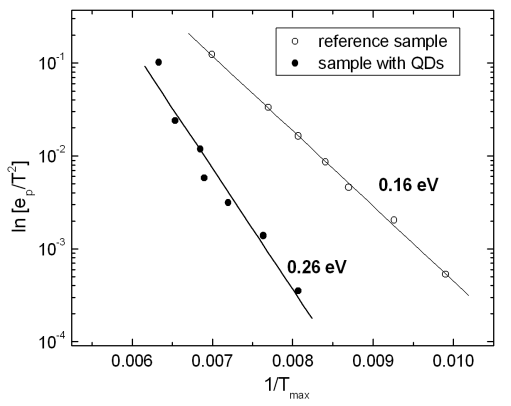

Fig. 3. Arrhenius plots for the reference sample (empty circles) and for the QD sample (full dots).

In Fig. 4a, b the DLTS scans taken during warming up the samples preceded by cooling down without an external bias and biased as in ordinary DLTS measurements are shown for comparison for both samples.

The way of cooling does not affect the DLTS signal for the reference sample. On the contrary, the DLTS signal related to the $0.26 \mathrm{eV}$ trap is observed solely if the DLTS measurements are preceded by the cooling down without bias. Such a behavior of DLTS signal was also observed by others for QD InAs/GaAs system [8, 9] and has been assigned either to the emission from the QDs themselves or from the defects at the interface. If the type-II band offset alignment observed for the bulk CdTe/ZnTe preserves for the studied here CdTe QDs embedded in ZnTe matrix, then the $0.26 \mathrm{eV}$ trap found by us for the QD sample cannot be assigned to the hole emission from QDs but it should be attributed either to the defects present in the wetting layer or $\mathrm{CdTe} / \mathrm{ZnTe}$ interface. However, it should be pointed out that due to variations in the energy induced by size and strain fluctuations from dot to dot for the self-assembled QD system, the energy obtained from the measurements can deviate strongly from 


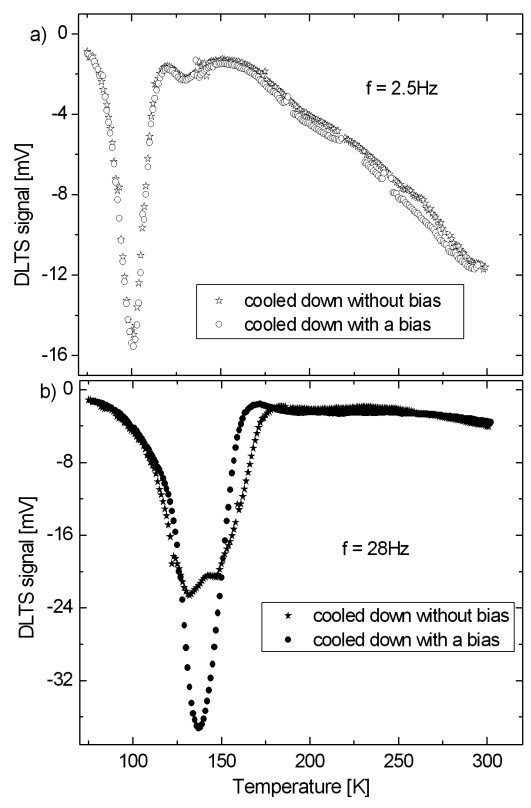

Fig. 4. DLTS spectra taken during warming up the samples preceded by cooling with or without external bias for (a) the reference- and (b) QD sample. In both cases $U_{\mathrm{R}}=-1.5 \mathrm{~V}$, and the pulse $U_{1}=0 \mathrm{~V}$ of $20 \mu \mathrm{s}$ width.

the bulk value. In order to find the origin of the $0.26 \mathrm{eV}$ further studies have to be performed.

\section{Conclusions}

The electrical properties of $\mathrm{CdTe} / \mathrm{ZnTe}-\mathrm{Al} / \mathrm{Ti}$ Schottky quantum dot structures were investigated by means of the capacitance-voltage $(C-V)$ measurements and DLTS. The object of the investigations was a sample with CdTe self-assembled QDs embedded in ZnTe (p-type) matrix. For a comparison a reference sample, in which QDs were absent, has been also examined. The $C-V$ characteristics exhibits a step corresponding to the discharging of QDs. The DLTS measurements for both samples yield the presence of a hole trap related to Zn vacancy with the thermal activation energy equal to $0.16 \mathrm{eV}$. The Arrhenius plot of the dominant peak found exclusively for the QDs sample reveals the thermal activation energy equal to $0.26 \mathrm{eV}$. Comparison of the results obtained for both samples leads to the conclusion that the signal corresponding to the peak for the QDs sample is presumably related to the hole emission from the defects located at the $\mathrm{CdTe}$ wetting layer or $\mathrm{CdTe} / \mathrm{ZnTe}$ interface.

\section{Acknowledgments}

The work has been financially supported by the Ministry of Science and Higher Education project No. N N202 091133

\section{References}

[1] D. Bimberg, M. Grundmann, N.N. Ledenstov, Quantum Dot Heterostructures, Wiley, Chichester 1999.

[2] H. Bellakhder, A. Outzourhit, E.L. Ameziane, Thin Solid Films 382, 30 (2001).

[3] K. Sato, S. Adachi, J. Appl. Phys. 73, 926 (1993).

[4] K. Sato, M. Hanafusa, A. Noda, A. Arakawa, M. Uchida, T. Asahi, O. Oda, J. Cryst. Growth 214/215, 1080 (2000).

[5] A. Continenza, S. Massidda, Phys. Rev. B 50, 11949 (1994).

[6] B. Späth, J. Fritsche, F. Säuberlich, A. Klein, W. Jaegermann, Thin Solid Films 480-481, 204 (2005).

[7] H. Tubota, Jpn. J. Appl. Phys. 2, 259 (1963).

[8] M.M. Sobolev, A.R. Kovsh, V.M. Ustinov, A.Yu. Egorov, A.E. Zhukov, Yu.G. Musikhin, Semiconductors 33, 157 (1999).

[9] M.M. Sobolev, I.V. Kochnev, V.M. Lantratov, N.A. Cherkashin, V.V. Emstev, Physica B 273-274, 959 (1999). 